\title{
Limited Unfolded Protein Response and Inflammation in Neuroserpinopathy
}

\author{
Irene López-González, MSc, Alberto Pérez-Mediavilla, MD, PhD, Marta Zamarbide, PhD, \\ Margarita Carmona, Tech, Benjamin Torrejón Escribano, Markus Glatzel, MD, \\ Giovanna Galliciotti, $\mathrm{PhD}$, and Isidre Ferrer, $\mathrm{MD}, \mathrm{PhD}$
}

\begin{abstract}
Familial encephalopathy with neuroserpin inclusion bodies (FENIB) is a rare disease characterized by the deposition of multiple intracytoplasmic neuronal inclusions that contain mutated neuroserpin. Tg-Syracuse (Tg-Syr) mice express Ser49Pro mutated neuroserpin and develop clinical and neuropathological features of human FENIB. We used 8-, 34-, 45- and 80-week-old Tg-Syr mice to characterize neuroinflammation and the unfolded protein response (UPR) in a neurodegenerative disease in which abnormal protein aggregates accumulate within the endoplasmic reticulum (ER). There were scattered neuroserpin inclusions in Tg-Syr mice at 8 weeks of age; the numbers of neurons involved and the amount of neuroserpin per neuron increased with age throughout the CNS to 80 weeks of age; no similar inclusions were found in wild type ( $\mathrm{Tg}-\mathrm{WT})$ mice at any age. Increases in numbers of astrocytes and microglia occurred at advanced disease stages. Among 22 markers in 80 -week-old Tg-Syr mice, only $I I I b$ and IIIOrb mRNAs in the somatosensory cortex and $\mathrm{CxClIO}$ and IllOrb mRNAs in the olfactory bulb were upregulated when compared with Tg-WT mice indicating a limited relationship between neuroserpin inclusions and inflammatory responses. The changes were accompanied by a transient increase in expression of Xbpl spliced at 45 weeks and increased ERdJ4 mRNAs at 80 weeks. The sequestration of UPR activators GRP78 and GRP94 in neuroserpin inclusions might
\end{abstract}

From the Institut de Neuropatologia, IDIBELL-Hospital Universitari de Bellvitge, Hospitalet de Llobregat (ILG, MC, IF), Barcelona, Spain; Cell and Molecular Neuropharmacology Laboratory, Neurosciences Division, Center for Applied Medical Research (CIMA), Department of Biochemistry and Genetics (APM, MZ), University of Navarra, Pamplona, Spain; IdiSNA, Navarre Institute for Health Research (APM, MZ), Pamplona, Spain; Unitat de Biologia, Centres Cientifics i Tecnològics, Universitat de Barcelona (BTE), Spain; Institute of Neuropathology, University Medical Center Hamburg-Eppendorf (MG, GG), Hamburg, Germany; Universitat de Barcelona, Hospitalet de Llobregat (IF), Barcelona, Spain; CIBERNED (Centro de Investigación Biomédica en Red de Enfermedades Neurodegenerativas) (IF), Spain.

Send correspondence to: Prof. I. Ferrer, Institut de Neuropatologia, Servei Anatomia Patologica, Hospital Universitari de Bellvitge, Carrer Feixa Llarga sn, 08907 Hospitalet de Llobregat, Barcelona, Spain; E-mail: 8082ifa@gmail.com

This study was funded by the Ministerio de Ciencia e Innovación, Instituto de Salud Carlos III - Fondos FEDER, a way to build Europe FIS grants PI14/00757 and PI14/00328. Funding for Giovanna Galliciotti: Deutsche Forschungsgemeinschaft (FG885) and the Werner Otto Foundation

Disclosure and conflict of interests: None. explain the limited UPR responses despite the accumulation of neuroserpin in the ER in this FENIB mouse model.

Key Words: Cytokines, Endoplasmic reticulum, Familial encephalopathy with neuroserpin inclusion bodies (FENIB), Inflammation, Microglia, Neuroserpin, Unfolded protein response.

\section{INTRODUCTION}

Neuroserpin (proteinase inhibitor 12, PI12 or SERPINI1) is an axonal-secreted serine protease inhibitor encoded by the serpin peptidase inhibitor, clade A, member 1: SERPINII gene in chromosome 3q26 (1-3). Neuroserpin forms covalent acylcomplexes in vitro with several proteases, such as tissue plasminogen activator and urokinase plasminogen inhibitor, whereas inhibition of plasmin and trypsin seems to be mediated by substrate-like interaction $(4,5)$. Neuroserpin is expressed in post-mitotic neurons during development in the olfactory bulb, cerebral neocortex, hippocampus, and amygdala at high levels (6), and in striatum, thalamus, brainstem, and spinal cord at lower levels in the adult (4). Neuroserpin is involved in neurite growth, synaptic plasticity, and regulation of the emotional state, and it is neuroprotective in stroke and seizures (7).

Mutations in neuroserpin gene cause familial encephalopathy with neuroserpin inclusion bodies ([FENIB]; OMIM \#604218) (8-16). Clinically, FENIB can manifest as slowly progressive dementia, dementia and epilepsy, and progressive myoclonus epilepsy (17). Neuropathologically, it is characterized by unique or multiple eosinophilic intracytoplasmic neuronal inclusions and isolated inclusions in the neuropil, called Collins bodies; these are strongly positive with periodic acid-Schiff (PAS) staining and resistant to diastase, and they contain aggregates of glycosylated mutant neuroserpin. Neuroserpin deposits are present in the cerebral neocortex, hippocampus, amygdala, striatum, thalamus, substantia nigra and other nuclei of the brainstem, spinal cord, and dorsal spinal ganglia, but the cerebellar cortex and inferior olives appear unaffected with the exception of one young patient with a severe disease course (13). A unique feature of FENIB when compared with other neurodegenerative diseases with abnormal protein aggregates is the localization of neuroserpin deposits within the endoplasmic reticulum (ER) as an amorphous or fine granular material often darker in the center 
$(9,10,12)$. Biochemically, these deposits are the result of abnormal mutated neuroserpin polymerization that produces sequences of linked monomers of the mutant protein that cannot be exported or degraded (18-21). An additional curious aspect of the disease is the limited microglial reaction when compared with other more common neurodegenerative diseases with abnormal protein aggregates. This is unexpected because ER localization of abnormal proteins induces ER stress, which is biochemically manifested as the unfolded protein response (UPR), and the UPR can initiate inflammation (22).

Two mouse models of FENIB have been generated by expressing the Syracuse (Ser49Pro) and Portland (Ser52Arg) neuroserpin mutations (23). Another line has been generated expressing the Gly392Glu mutation in neuroserpin (24). A rat model of human FENIB has been created by overexpressing megsin, a novel member of the serpin family (25). Although this model resembles the neuropathological features of human neuroserpinopathy, megsin has never been described in human FENIB. In contrast, Tg-Syracuse ( $\mathrm{Tg}$-Syr), $\mathrm{Tg}$ Portland and Tg-G392E reproduce clinical, neuropathological, and biochemical traits of human FENIB, including neuroserpin deposits in the ER (and lysosomes in Tg-G392E); moreover, neuroserpin inclusions appear much earlier than the clinical signs. Therefore, these models are particularly useful to investigate mechanisms of neurodegeneration with disease progression $(23,25)$.

The present study was designed to learn about putative inflammatory responses, or the relative lack of them, in the Tg-Syr model of FENIB as a part of a larger comparative study of inflammation and mechanisms involved in human neurodegenerative diseases and corresponding mice models. These include APP/PS1 Tg mice bearing the Swedish mutation in APP and PSEN1 deletion used as a model of $\beta$-amyloidopathy mimicking Alzheimer disease (AD), P301S-MAPT mouse model of tauopathy, and murine Creutzfeldt-Jakob disease (CJD), produced in Prp $\mathrm{mo}^{-/-}$, PrP hum ${ }^{+/+}$mice infected with brain homogenates from CJD type MM1 (26-28). The major interest in comparing neuroserpinopathy with other neurodegenerative diseases with abnormal protein aggregates is based on the accumulation of the abnormal neuroserpin within the ER and the apparent discrete data regarding inflammatory responses in FENIB.

\section{MATERIALS AND METHODS}

\section{Animals}

The experiments were carried out in transgenic mice overexpressing S49P-Syracuse human neuroserpin (S49PSyracuse: Tg-Syr) and wild-type human neuroserpin ( $\mathrm{Tg}$ WT) aged $8,22-25,34,45$, and 80 weeks $(n=3$ mice for each phenotype and age). The generation of transgenic mice has been reported elsewhere (23).

All animal procedures were performed in accordance with the institutional guidelines of the animal facility of the University Medical Center Hamburg-Eppendorf, Hamburg, Germany. Mice brains were dissected and either fixed for 16 hours in $4 \%$ paraformaldehyde in phosphate-buffered saline
(pH 7.5) for subsequent immunohistochemistry or immediately frozen with dry ice and stored at $-80^{\circ} \mathrm{C}$ for subsequent RNA purification.

\section{Histology and Immunohistochemistry}

Fixed brain tissue samples from Tg-WT and Tg-Syr mice aged $8,22-25,34,45$, and 80 weeks were embedded in paraffin, and coronal $4-\mu \mathrm{m}$-thick sections were obtained with a sliding microtome. De-waxed sections were stained with hematoxylin and eosin and PAS stains and processed for immunohistochemistry. The sections were boiled in citrate buffer for 20 minutes to enhance antigenicity. Endogenous peroxidases were blocked with $10 \%$ methanol- $1 \% \quad \mathrm{H}_{2} \mathrm{O}_{2}$ solution for 15 minutes, followed by $3 \%$ normal horse serum solution, and then incubated at $4{ }^{\circ} \mathrm{C}$ overnight with one of the following primary antibodies: anti-glial fibrillary acidic protein ([GFAP], rabbit 1:250; Z0334, Dako, Glostrup, Denmark), microglia (anti-Iba1, rabbit 1:250; 019-19741, Wako, Richmond, VA), and anti-neuroserpin (polyclonal antibodies generated by genetic immunization by the Antibody Core Facility, University Medical Center Hamburg-Eppendorf, used at dilutions of 1:100 and 1:200) (29). Sections were subsequently rinsed and incubated with biotinylated secondary antibody (Dako), followed by EnVision + system peroxidase (Dako) and, finally, chromogen DAB (diaminobenzidine) and $\mathrm{H}_{2} \mathrm{O}_{2}$. Some sections were incubated without the primary antibodies; no immunostaining was detected in these sections. Sections were lightly counterstained with hematoxylin. After staining, the sections were dehydrated and coverslipped for microscopic assessment.

For double-labeling immunofluorescence, de-waxed sections were treated with citrate buffer for 20 minutes to enhance antigenicity and then stained with a saturated solution of Sudan black B (Merck Millipore, Darmstad, Germany) for 30 minutes to block lipofuscin autofluorescence. Immediately afterwards, the sections were rinsed in $70 \%$ ethanol and washed in distilled water. After blocking endogenous peroxidases with $10 \%$ fetal bovine serum for 90 minutes, sections from Tg-WT and Tg-Syr mice aged 34, 45 and 80 weeks were incubated with anti-neuroserpin mouse monoclonal antibodies and one of the following antibodies: glucose-regulated protein 78 (GRP78, rabbit, dilution 1:800; ab21685, Abcam, Cambridge, UK); glucose-regulated protein 94 (GRP94, rabbit dilution 1:1,000, ab3674, Abcam); anti-inositol-requiring enzyme 1 (IRE1) (phospho S724) (IRE1-P, rabbit 1:500; ab48187, Abcam); and X-box binding protein ([XBP1], rabbit 1:500; ab37152, Abcam, which recognizes the non-spliced shorter form of XBP1) and then incubated with Alexa488 or Alexa546 fluorescence secondary antibodies against the corresponding host species (1:400, Molecular Probes, Eugene, OR). Nuclei were stained with DRAQ5 ${ }^{\mathrm{TM}}$ (1:2,000, Biostatus, Leicestershire, UK). After washing, the sections were mounted in Immuno-Fluore mounting medium (ICN Biomedicals, Irvine, CA), sealed, and dried overnight. Sections were examined with a Leica TCS-SL confocal microscope (Leica, Wetzlar, Germany). In addition, to block autofluorescence of possible lipofuscin granules by pre-incubating the tissue sections with Sudan black, non-specific 
autofluorescence was controlled by incubating a few sections without primary antibodies. No immunostaining was observed in these sections.

Astrocyte (GFAP-positive) and microglia (Iba1-positive) cells in the somatosensory cortex, amygdala, thalamus, hippocampus, and brainstem were counted in 3 representative fields per region in serial non-consecutive sections at the ages of 22$25,34,45$, and 80 weeks using a microscope Olympus BX50 (Shinjuku, Tokyo, Japan) at a magnification x200. Three animals per group were used for quantifications; selected areas were similar in all cases and measured $0.115 \mathrm{~mm}^{2}$.

\section{RNA Purification}

Total RNA from olfactory bulb and neocortex of Tg-Syr and Tg-WT mice aged $8,22-25,34,45$, and 80 weeks $(n=3$ per group) was isolated with the Rneasy Lipid Tissue Mini Kit (Qiagen, Hilden, Germany) following the manufacturer's protocol. RNA concentration of each sample was measured using a NanoDrop Spectrophotometer (Thermo Fisher Scientific, Wilmington, DE). RNA integrity was tested using the Agilent 2100 BioAnalyzer (Agilent Technologies, Palo Alto, CA). In addition, the thalamus was available only in $\mathrm{Tg}$-Syr and $\mathrm{Tg}$ WT mice aged 45 weeks $(n=3$ per phenotype) and was processed in the same way. RNA integrity number values were higher than 8.5 in every sample.

\section{TaqMan Quantitative RT-PCR}

cDNA was prepared using the High-Capacity cDNA Reverse Transcription kit (Applied Biosystems, Foster City, CA) following the protocol provided by the supplier. Parallel reactions for each RNA sample were run in the absence of MultiScribe Reverse Transcriptase to assess the lack of contamination of genomic DNA.

TaqMan quantitative reverse transcription-polymerase chain reaction (RT-PCR) assays were performed in duplicate for each gene on cDNA samples in 384-well optical plates using an ABI Prism 7900 Sequence Detection system (Applied Biosystems, Life Technologies, Waltham, MA). For each 10 $\mu \mathrm{L}$ TaqMan reaction, $4.5 \mu \mathrm{L}$ cDNA was mixed with $0.5 \mu \mathrm{L}$ 20x TaqMan Gene Expression Assays and $5 \mu \mathrm{L}$ of $2 \mathrm{x}$ TaqMan Universal PCR Master Mix (Applied Biosystems). The identification numbers and names of TaqMan probes are shown in Table 1. Hypoxanthine-guanine phosphoribosyltransferase (Hprt), alanyl-transfer RNA synthetase (Aars), and X-prolyl aminopeptidase (aminopeptidase P) 1 (Xpnpepl) were used as housekeeping genes for normalization.

The reactions were carried out using the following parameters: $50^{\circ} \mathrm{C}$ for 2 minutes, $95^{\circ} \mathrm{C}$ for 10 minutes, and 40 cycles of $95^{\circ} \mathrm{C}$ for 15 seconds and $60^{\circ} \mathrm{C}$ for 1 minute. Finally, all TaqMan PCR data were captured using the Sequence Detection Software (SDS version 2.2.2, Applied Biosystems).

ER stress sensors were quantified by SYBR green quantitative RT-PCR. Two $\mu \mathrm{g}$ of total RNA was used as a template to synthesize cDNA with the SuperScript III FirstStrand Synthesis System for RT-PCR (Invitrogen, Life Technologies, Carlsbad, CA). Real-time quantitative PCR assays were then performed in triplicate on these cDNAs in the presence of the PCR Master Mix (Power SYBRGreen, Applied Biosystems) to detect the amplification products. Samples were analyzed simultaneously for ribosomal protein 36B4 mRNA as an internal control using an ABI Prims 7300 sequence detector (Applied Biosystems). The reactions were carried out using the following parameters: $50^{\circ} \mathrm{C}$ for 2 minutes, $95^{\circ} \mathrm{C}$ for 10 minutes, and 40 cycles of $95^{\circ} \mathrm{C}$ for 10 seconds, $60^{\circ} \mathrm{C}$ for 15 seconds, and $72^{\circ} \mathrm{C}$ for 25 seconds. Finally, all SYBR green PCR data were captured using the Sequence Detection Software (SDS version 2.2.2, Applied Biosystems). Forward and reverse primer sequences for quantitative PCR are indicated in Table 1.

Samples were analyzed with the double-delta cycle threshold $(\Delta \Delta \mathrm{CT})$ method using 8-week-old Tg-WT mouse samples as controls. Values of the 3 housekeeping genes were similar; values of inflammation-related genes were normalized using the mean values of the 3 housekeeping genes. Results were analyzed with two-way ANOVA followed by Tukey post hoc or Student t-test when required. The significance level was set at $* \mathrm{p}<0.05, * * \mathrm{p}<0.01$, and $* * * \mathrm{p}<0.001$.

\section{RESULTS}

\section{Histological and Immunohistochemical Observations}

Neuroserpin inclusions, as revealed with the anti-neuroserpin antibody, were observed in scattered neurons of the neocortex, hippocampus, amygdala, thalamus, and brainstem in $\mathrm{Tg}$ Syr mice at the age of 8 weeks. The numbers of affected neurons and the amount of neuroserpin inclusions increased with disease progression: low at the age of 34 weeks and increasing thereafter mainly in the olfactory bulb, neocortex, amygdala, and brainstem in $\mathrm{Tg}$-Syr mice aged 45 weeks, and dramatically in all regions in mice aged 80 weeks (Fig. 1), as detailed in the seminal description of this murine model (23). No inclusions were seen in Tg-WT mice at any age (data not shown).

Glial responses were examined with anti-GFAP antibodies to label astrocytes, antibody IBA1 to identify microglia, and PAS staining in consecutive serial sections which included the cerebral neocortex, hippocampus, amygdala, thalamus and brainstem in Tg-Syr and Tg-WT littermates at different ages. Astrocyte and microglia populations were similar in Tg-WT and Tg-Syr mice despite the progressive accumulation of Collins bodies revealed by PAS staining in Tg-Syr mice. This was even observed at advanced stages of the disease in the cerebral neocortex, hippocampus, amygdala, and brainstem, but, curiously, not in the thalamus, where the number of astrocytes and microglia was significantly higher in Tg-Syr when compared with Tg-WT littermates (Fig. 2).

Quantitative studies showed regional differences in the number of astrocytes in Tg-WT and Tg-Syr mice; the hippocampus was enriched in astrocytes at any age followed by the brainstem when compared with the amygdala, thalamus, and somatosensory cortex. An increased number of astrocytes was observed in Tg-WT and Tg-Syr at the age of 80 weeks. The major difference between phenotypes was found in the thalamus where the number of astrocytes was higher in $\mathrm{Tg}-\mathrm{Syr}$ when compared with Tg-WT mice (Table 2). 
TABLE 1. TaqMan Probes and PCR Primers Used for Study of Murine Cytokine and Immune Response Mediator Expression

\begin{tabular}{|c|c|c|}
\hline Symbol & Gene Name & TaqMan and Primer Sequences \\
\hline Hprt & Hypoxanthine-guanine phosphoribosyltransferase & CAGCAGTGAGGACAAAACCGAGTTT \\
\hline Aars & Alanyl-tRNA synthetase & GGACTGATTATGGACAGGACTGAAA \\
\hline Clql1 & Complement component 1 , q subcomponent 1 & AACGGCCAGGTGCGGGCCAGTGCAA \\
\hline Clqtnf7 & $\mathrm{C} 1 \mathrm{q}$ and tumor necrosis factor related protein 7 & AAAGGGCACTGCAGGTCTAAAAGGT \\
\hline C3arl & Complement component 3 a receptor 1 & GTGTGCTTGACTGAGCCATGGAGTC \\
\hline$C 4 b$ & Complement component $4 \mathrm{~b}$ & GACATGAGCAAGGTCTTTGAAGTAA \\
\hline Csflr & Colony stimulating factor 1 receptor & CTAAAAACTGCATCCACCGGGACGT \\
\hline Csf $3 r$ & Colony stimulating factor 1 receptor & GCTACTCCCCAGAAGTCTGGAGAGC \\
\hline Tlr4 & Toll-like receptor 4 & CCCTGCATAGAGGTAGTTCCTAATA \\
\hline $\operatorname{Tlr} 7$ & Toll-like receptor 7 & CCCTGCATAGAGGTAGTTCCTAATA \\
\hline Ccl3 & Chemokine (C-C motif) ligand 3 & GTCTTCTCAGCGCCATATGGAGCTG \\
\hline Ccl4 & Chemokine (C-C motif) ligand 4 & GTTCTCAGCACCAATGGGCTCTGAC \\
\hline $\mathrm{CxCl10}$ & Chemokine (C-X-C motif) ligand 10 & GACTCAAGGGATCCCTCTCGCAAGG \\
\hline$I l 1 b$ & Interleukin $1 \beta$ & GACCCCAAAAGATGAAGGGCTGCTT \\
\hline Il6 & Interleukin 6 & TGAGAAAAGAGTTGTGCAATGGCAA \\
\hline Il6st & Interleukin 6 signal transducer & ACCCACTTGAGAGGACGCCTCCTGG \\
\hline Tnfa & Tumor necrosis factor $\alpha$ & GCCCACGTCGTAGCAAACCACCAAG \\
\hline Tnfrsfla & Tumor necrosis factor receptor superfamily member 1a & CTTGCAGCCACTGCAAGAAAAATGA \\
\hline Illo & Interleukin 10 & GAAGACTTTCTTTCAAACAAAGGAC \\
\hline Illora & Interleukin 10 receptor $\alpha$ & TATCACGACGGAGCAGTATTTCACT \\
\hline IllOrb & Interleukin 10 receptor $\beta$ & CAGGCAATGACGAAATAACCCCTTC \\
\hline $\operatorname{Tgbl}$ & Transforming growth factor $\beta 1$ & CTGAACCAAGGAGACGGAATACAGG \\
\hline$T g f b 2$ & Transforming growth factor $\beta 2$ & TCGAGGCGAGATTTGCAGGTATTGA \\
\hline$C H O P F w$ & $\mathrm{C} / \mathrm{EBP}-$ homologous protein forward & GCTGGGAGCTGGAAGCCTGGTATG \\
\hline CHOP Rev & $\mathrm{C} / \mathrm{EBP}$-homologous protein reverse & TCCCTGGTCAGGCGCTCGATTTCC \\
\hline$G p r 78 F w$ & Glucose-regulated protein $78 \mathrm{kDa}$ forward & ACCAACTGCTGAATCTTTGGAAT \\
\hline Gpr78 Rev & Glucose-regulated protein $78 \mathrm{kDa}$ reverse & GAGCTGTGCAGAAACTCCGGCG \\
\hline$E R d j 4 F w$ & Endoplasmic reticulum-localized DnaJ 4 forward & GAAAACTCCTGGAAGTGATGCCTTTGTCTA \\
\hline ERdj4 Rev & Endoplasmic reticulum-localized DnaJ 4 reverse & TCACAAATTAGCCATGAAGTACCACCCTGA \\
\hline$X b p l F w$ & X-box binding protein 1 forward & CCCCACTGACAGAGAAAGGGAGG \\
\hline Xbpl Rev & $\mathrm{X}$-box binding protein 1 reverse & GCAGGTGCAGGCCCGATTGTCAC \\
\hline Xbpl splicing Rev & $\mathrm{X}$-box binding protein 1 splicing reverse & CGGGTCTGCTGAGTCCGCAGCAG \\
\hline $36 B 4 F w$ & Acidic ribosomal phosphoprotein $P 0$ forward & ААСАТСТСССССТТСТССТТ \\
\hline $36 B 4 \operatorname{Rev}$ & Acidic ribosomal phosphoprotein $P 0$ reverse & GAAGGCCTTGACCTTTTCAG \\
\hline
\end{tabular}

Quantitative studies of microglia revealed an increase in the number of microglial cells in the thalamus in Tg-Syr mice when compared with Tg-WT aged 45 weeks. Microglial cells increased in number whatever the phenotype in mice aged 80 weeks. However, the number of microglia was higher in the thalamus of Tg-Syr mice when compared with corresponding Tg-WT mice aged 80 weeks (Table 2).

\section{Gene Expression of Cytokines and Mediators of the Immune Response}

Gene expression levels of cytokines and mediators of the immune response in Tg-WT and Tg-Syr mice in the olfactory bulb and neocortex were assessed in mice aged 8, 22-25,
34, 45, and 80 weeks. Modifications were related to aging and phenotype.

In Tg-WT mice, the expression of Clqtnf7, C3arl, C4b, Csf3r, Tlr7, Ccl3, Ccl4, Ccl6, CxCl10, and Tnfa mRNAs was significantly increased in the neocortex with age (Table 3). Increased expression of Clqtnf7, C4b and Ccl4 mRNAs, and decreased expression of $T g f b 2$ mRNA were observed in the olfactory bulb with age (Table 4 ).

Regarding Tg-Syr mice, C3arl, C4b, Csf3r, Ccl3, Ccl4, Ccl6, CxCl10, Illb, Il6st, Tnfa, IllOrb, and Tgfbl mRNAs were significantly increased in the neocortex with age, whereas C4b, Csf3r, Ccl4, Cxcllo, Tnfa, and Illora mRNAs were significantly increased in the olfactory bulb with age (Tables 3, 4). 


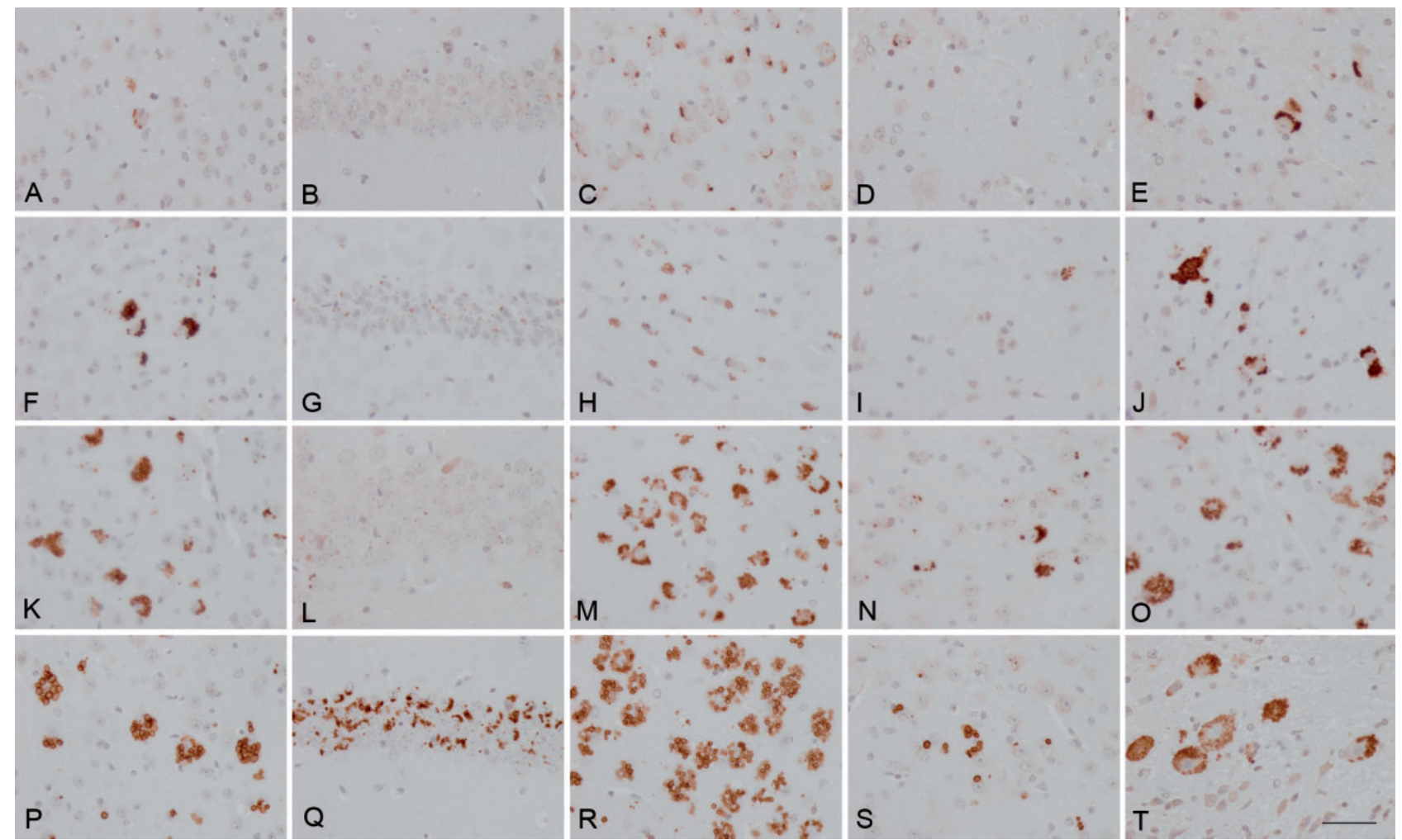

FIGURE 1. Neuroserpin immunohistochemistry in Tg-Syr mice in the cerebral neocortex (A, F, K, P), CA1 region of the hippocampus (B, G, L, Q), amygdala (C, H, M, R), thalamus (D, I, N, S) and brainstem (E, J, O, T) at 8 weeks (A-E), 34 weeks (F-J), 45 weeks (K-0) and 80 weeks (P-T) of age. Neuroserpin inclusions were scarce at the age of 8 weeks but the numbers of neurons affected and the numbers of neuroserpin inclusions per neuron increase with disease progression mainly in the cerebral neocortex, amygdala and brainstem at the age of 45 weeks and in all regions at the age of 80 weeks. Note that the thalamus is the region with the fewest neuroserpin inclusions. Paraffin sections, slightly counterstained with hematoxylin. Scale bar $=25 \mu \mathrm{m}$.

In contrast with the substantial and wide modifications with age in both Tg-Syr and Tg-WT animals, only minor differences were found when comparing gene expression in $\mathrm{Tg}$ Syr and Tg-WT littermates. Ccl6 mRNA was transiently increased $(\mathrm{p}<0.05)$ in the neocortex of $\mathrm{Tg}$-Syr mice aged 8 weeks when compared with Tg-WT, whereas Illb and IllOrb were increased $(\mathrm{p}<0.05$ and $\mathrm{p}<0.01$, respectively) in $\mathrm{Tg}$ Syr aged 80 weeks when compared with Tg-WT (Table 3 ). Transient decrease in Ill0ra mRNA $(\mathrm{p}<0.01)$ and Clqtnf7 $(\mathrm{p}<0.05)$ was found in the olfactory bulb in Tg-Syr mice aged 34 weeks and 45 weeks, respectively, when compared with Tg-WT littermates. Only $\mathrm{CxCl10}$ and $1110 \mathrm{rb}$ mRNAs were upregulated ( $\mathrm{p}<0.01$ and $\mathrm{p}<0.05$, respectively) in the olfactory bulb in Tg-Syr mice aged 80 weeks when compared with Tg-WT (Table 4).

Samples of the thalamus were available in Tg-Syr and Tg-WT mice aged 45 weeks. Only Il10 was significantly upregulated in Tg-Syr when compared with Tg-WT $(3.15 \pm 0.17$ vs $1.00 \pm 0.28$, respectively, $\mathrm{p}<0.01$ ).

\section{Markers of the UPR}

The mRNA expression levels of targets of the 3 UPR pathways, PKR-like ER kinase (PERK): CHOP-10 (CHOP, chop) and Asns; activating transcription factor 6 (ATF6): Grp78 and total Xbpl; and IRE1: ERdJ4 and spliced Xbpl were examined in Tg-Syr and Tg-WT aged 34, 45 and 80 weeks (Fig. 3A). Xbpl spliced was significantly transiently increased $(\mathrm{p}<0.05)$ in $\mathrm{Tg}$-Syr mice at the age of 45 weeks, whereas ERdJ4 was significantly increased $(\mathrm{p}<0.001)$ at the age of 80 weeks (Fig. 3B).

\section{Double-Labeling Immunofluorescence and Confocal Microscopy of Neuroserpin and Markers of the UPR}

Double-labeling immunofluorescence disclosed that practically all neuroserpin inclusions colocalized GRP78 and GRP94 in Tg-Syr mice aged 45 and 80 weeks in the neocortex, amygdala, and brainstem (Fig. 4). No neuroserpin colocalization was seen with anti-IRE1 (phospho S724) and XBP1 antibodies (data not shown).

\section{DISCUSSION}

\section{Neuropathology of Tg-Syr}

Neuropathological characteristics of disease progression in the present study are the same as those already 


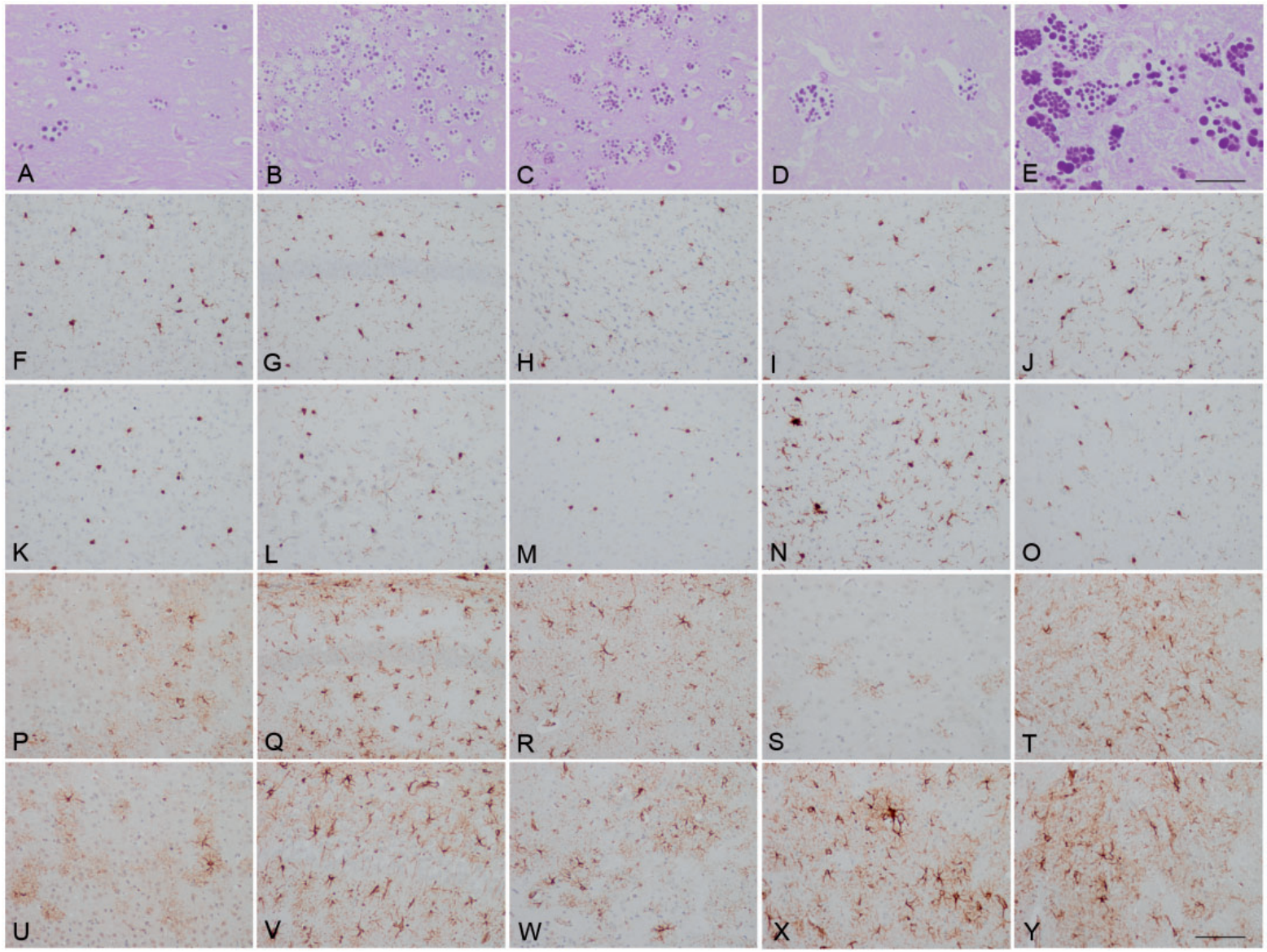

FIGURE 2. Comparative aspects of glial responses in Tg-Syr and Tg-WT mice at the age of 80 weeks. Collins bodies were revealed with PAS staining (A-E), astrocytes were demonstrated with anti-GFAP immunohistochemistry (F-0), and microglia were demonstrated with anti-lba1 IHC (P-Y) in Tg-Syr (A-E, K-O, U-Y) and Tg-WT (F-J, P-T) mice in the cerebral neocortex (A, F, $\mathbf{K}, \mathbf{P}, \mathbf{U})$, CA1 region of the hippocampus $(\mathbf{B}, \mathbf{G}, \mathbf{L}, \mathbf{Q}, \mathbf{V})$, amygdala $(\mathbf{C}, \mathbf{H}, \mathbf{M}, \mathbf{R}, \mathbf{W})$, thalamus $(\mathbf{D}, \mathbf{I}, \mathbf{N}, \mathbf{S}, \mathbf{X})$ and brainstem $(\mathbf{E}, \mathbf{J}, \mathbf{O}, \mathbf{T}, \mathbf{Y})$. No differences in glial responses between Tg-Syr and Tg-WT mice were seen in the cerebral neocortex, CA1 region of the hippocampus, amygdala and brainstem; however, astrocytes and microglia were more numerous in the thalamus in Tg-Syr mice. Note that the greatest glial responses in the thalami do not parallel with the number of Collins bodies in this region. Paraffin sections; scale bars, A-E, $25 \mu \mathrm{m} ; \mathbf{F}-\mathbf{Y}, 50 \mu \mathrm{m}$.

TABLE 2. Quantification of Astrocytes and Microglia in Brain Regions of Tg-Syr (Syr) and Tg-WT Mice Aged 22-25, 34, 45, and 80 Weeks

\begin{tabular}{|c|c|c|c|c|c|c|c|c|c|c|c|c|c|c|c|c|}
\hline & \multicolumn{8}{|c|}{ GFAP + Cells } & \multicolumn{8}{|c|}{ Iba-1 + Cells } \\
\hline & \multicolumn{2}{|c|}{$22-25 w$} & \multicolumn{2}{|l|}{$34 \mathrm{w}$} & \multicolumn{2}{|l|}{$45 w$} & \multicolumn{2}{|l|}{$80 w$} & \multicolumn{2}{|c|}{$22-25 w$} & \multicolumn{2}{|l|}{$34 w$} & \multicolumn{2}{|l|}{$45 w$} & \multicolumn{2}{|l|}{$80 \mathrm{w}$} \\
\hline & WT & Syr & WT & Syr & WT & Syr & WT & Syr & WT & Syr & WT & Syr & WT & Syr & WT & Syr \\
\hline Somatosensory cortex & 1 & 1 & 1 & 1 & 1 & 1 & 2 & 2 & 2 & 2 & 2 & 2 & 2 & 2 & 4 & 4 \\
\hline Amygdala & 2 & 2 & 2 & 2 & 2 & 2 & 3 & 3 & 2 & 2 & 2 & 3 & 2 & 2 & 4 & 4 \\
\hline Thalamus & 1 & 1 & 1 & 1 & 1 & 1 & 2 & 4 & 2 & 2 & 2 & 2 & 2 & 4 & 4 & 5 \\
\hline
\end{tabular}

GFAP+, glial fibrillary acidic protein-positive cells (astrocytes); Iba-1+, Iba-positive cells (microglia); Tg-Syr, Tg-Syracuse mice; Tg-WT, Tg-wild type mice; w, weeks; WT, wild type.

Values represent the number of cells per $\mathrm{mm}^{2}: 1<30 ; 2: 30-70 ; 3: 70-120 ; 4: 120-150 ; 5: 150-200$. Major differences in the number of astrocytes and microglia are seen between the ages of 45 and 80 weeks regardless phenotype. An increase in the number of astrocytes is found in the thalamus of Tg-Syr at the age of 80 weeks and especially of microglia in the thalamus of Tg-Syr at the age of 45 and 80 weeks. 


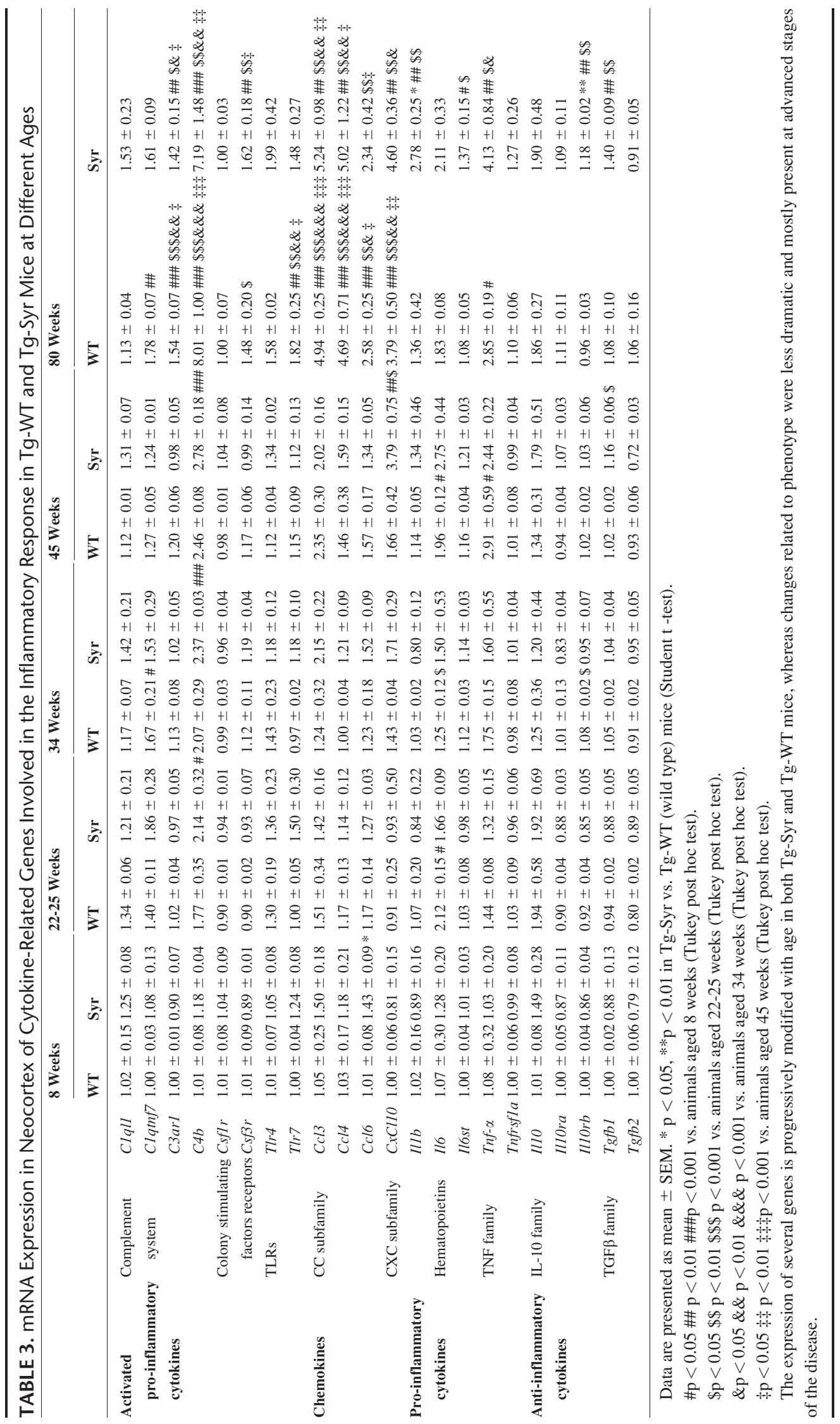




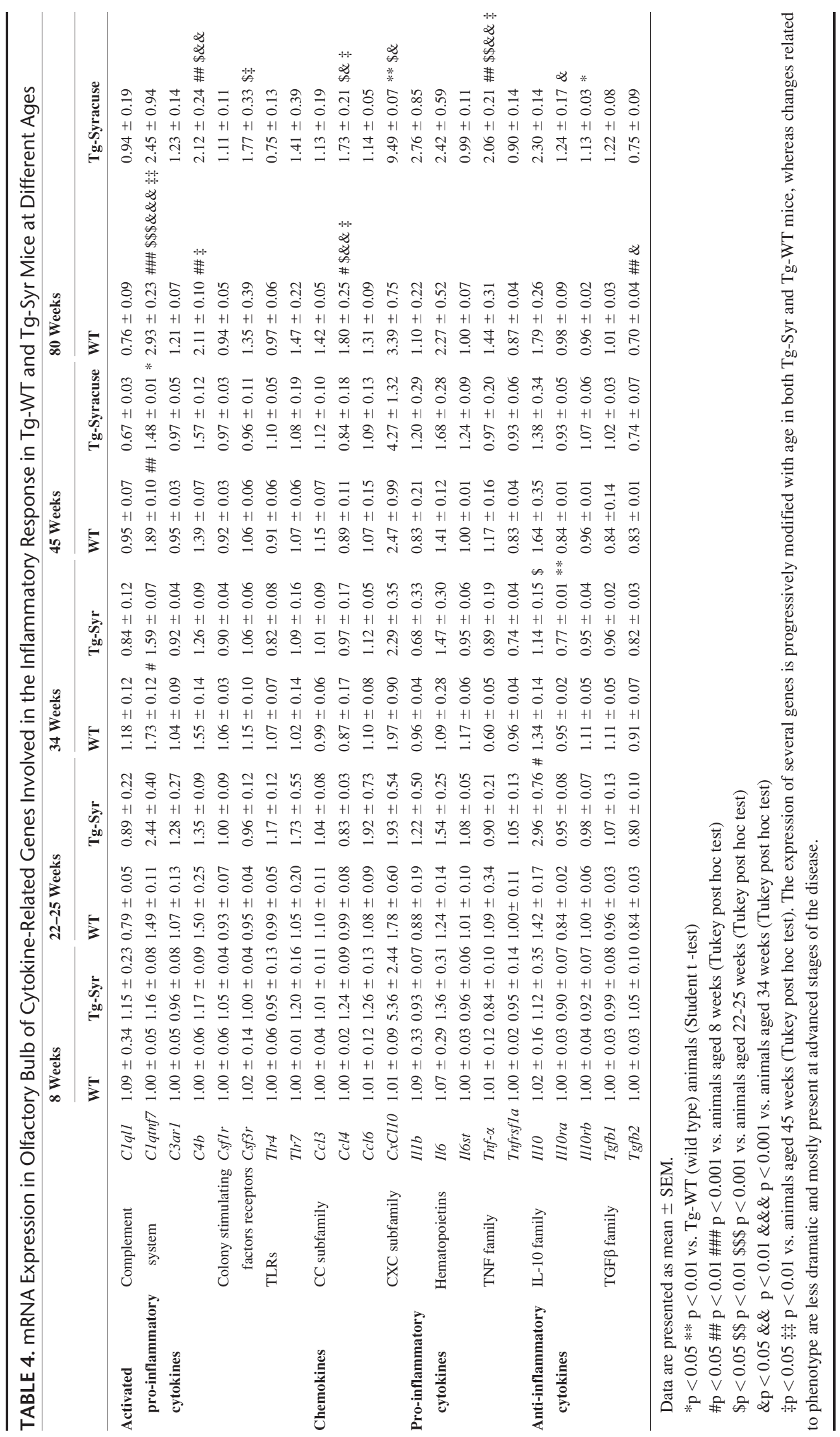



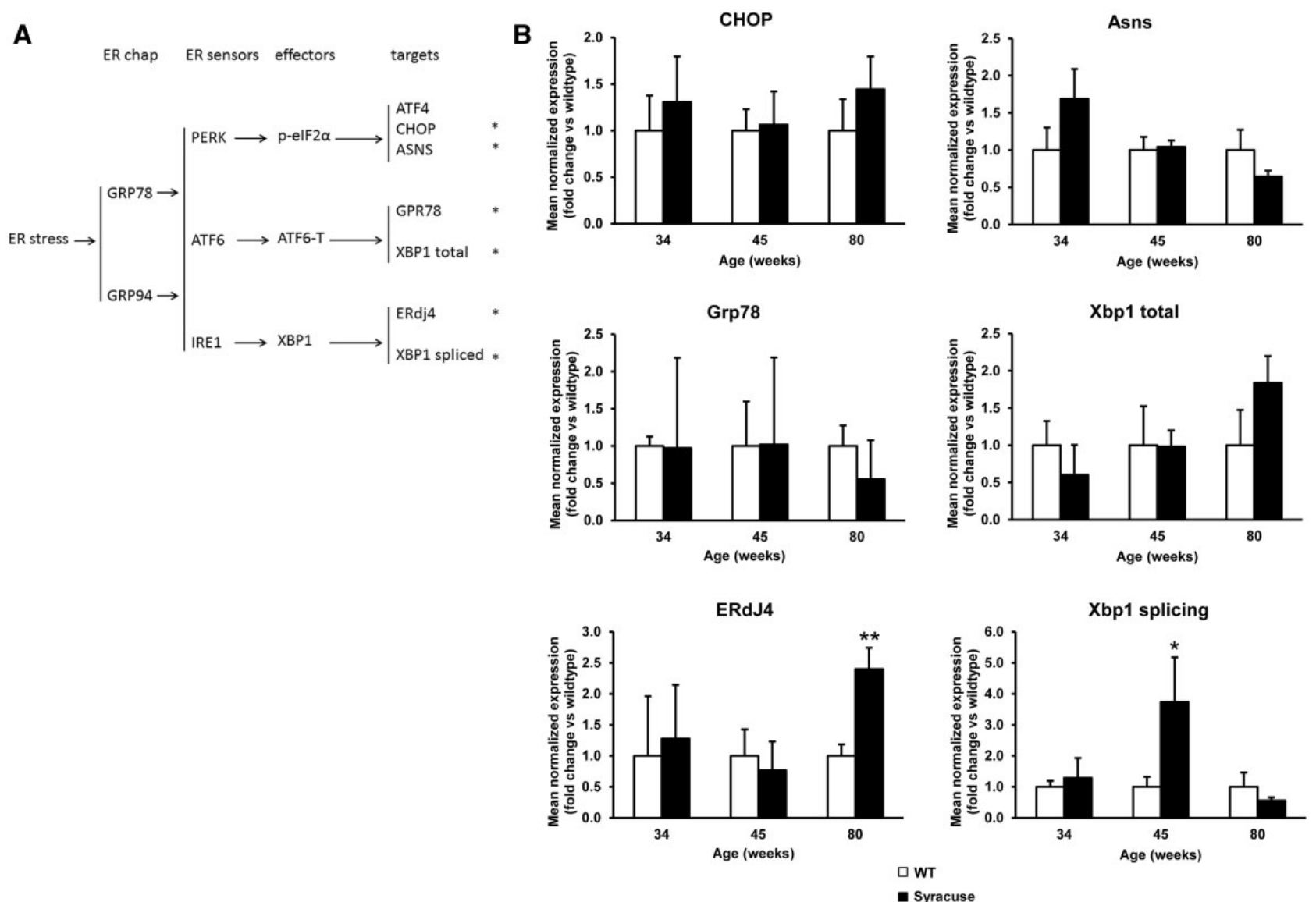

FIGURE 3. (A) Scheme of the ER chaperones (ER chap: inducers), GRP78 (glucose-related protein 78 kDa), and GRP94 (glucoserelated protein $94 \mathrm{kDa}$ ), 3 major ER stress sensors (ER sensors): PERK (PKR-like ER kinase), ATF6 (activating transcription factor 6) and IRE1 (inositol-requiring enzyme 1 (IRE1); corresponding downstream effectors (effectors): p-elF2 $\alpha$ (phosphorylated eukaryotic initiation factor $2 \alpha$ ), ATF6-T: ATF6-truncated and XBP-1, and targets tested (*) CHOP (C/EBP-homologous protein), ASNS (asparagine synthase), GRP78, XBP1 (X-box binding protein 1), ERdj4 (endoplasmic reticulum-localized Dnal 4) and XBP1 splicing. (Figure is modified from Zamarbide M, Martinez-Pinilla E, Ricobaraza A, Aragón T, et al. PLoS One 2013;8:e71082) (30). (B) mRNA expression of CHOP, Asns, Gpr78, Xbp1, ERdj4 and spliced Xbp1 in Tg-Syr and wild type (WT) mice at the ages of 34, 45 and 80 weeks. Xbp1 splicing is transiently increased at 45 weeks and ERdj4 at 80 weeks; p-elF2 $\alpha$ (eukaryotic translation initiation factor 2A phosphorylated), ATF4 (activating transcription factor 4); ${ }^{*} \mathrm{p}<0.05 ;{ }^{* * *} \mathrm{p}<0.001$.

reported for the Tg-Syr line $(23,31)$. These include the presence of single and multiple PAS-positive, neuroserpin-immunoreactive intracytoplasmic inclusions principally in neurons of the olfactory centers, cerebral neocortex, amygdala, hippocampus and brainstem, and in lesser numbers in the thalamus. A few inclusions are present at the age of 8 weeks, and the number of neurons affected and of inclusions increases with disease progression.

\section{Inflammation Markers in Tg-Syr}

Gene expression of cytokines and mediators of the immune response is strikingly discrete when compared with the very large extent of gene deregulation of the same cytokines and mediators in murine models of $\mathrm{AD}$, tauopathy, and CJD (26-28), particularly considering that those studies were carried out with the same probes by the same researchers in our laboratory. It can be argued that modest differences between phenotypes may be due to the small number of animals studied at every time point. However, significant differences were identified with age in Tg-WT and Tg-Syr in the present series as already demonstrated in mice during normal aging and in aged $\mathrm{Tg}$ mice assessed so far (26-28). Therefore, changes in Tg-Syr are not the mere expression of accelerated aging but rather are independent. Moreover, gene deregulation of cytokines and mediators of the immune response is discrete in Tg-Syr mice. This is in line with the limited astrocyte and microglia reaction in these mice when compared with AD, tauopathy, and CJD murine models (26-28).

Furthermore, inflammation markers do not correlate with the number of neuroserpin inclusions through disease progression. The thalamus is a good example of this because microglia were significantly increased at 45 and 80 weeks and $I l 10$ was upregulated at least at 45 weeks (the only time 


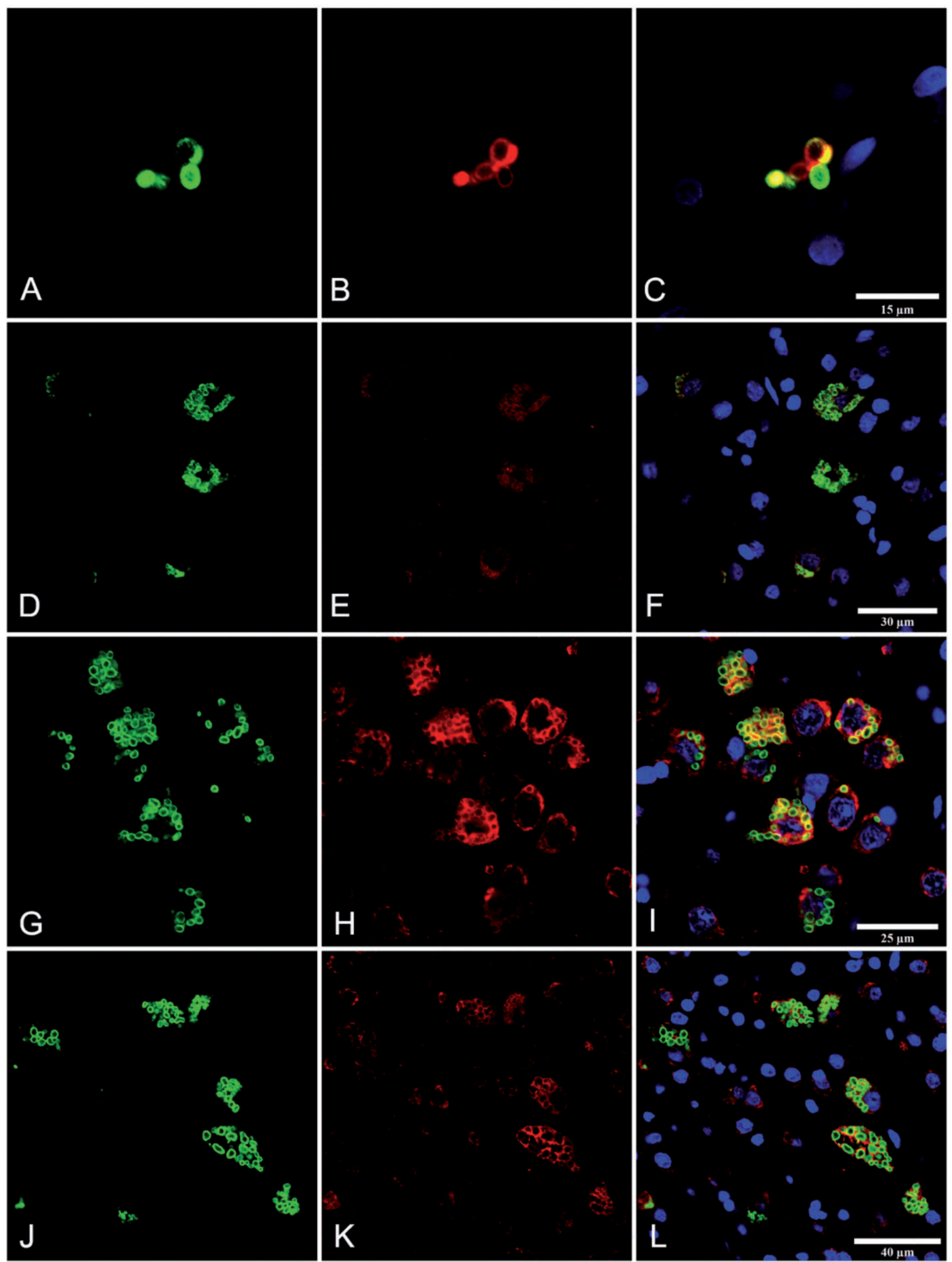

FIGURE 4. Double-labeling immunofluorescence demonstrating neuroserpin (green) and GRP78 (red) (A-C, G-I), and neuroserpin (green) and GRP94 (red) (D-F, J-L) in the brainstem of Tg-Syr mice aged 45 weeks (A-F) and 80 weeks (G-L). Note colocalization of both chaperones with neuroserpin inclusions (merge: $\mathbf{C}, \mathbf{F}, \mathbf{I}, \mathbf{L})$. Paraffin sections; nuclei stained with $\mathrm{DRAQ5}^{\mathrm{TM}}$ (blue); C, E, merge; scale bar $=20 \mu \mathrm{m}$. 
point available for mRNA studies in the thalamus) in Tg-Syr despite the relatively limited number of neuroserpin inclusions. Ccl6 mRNA is transiently increased in the neocortex of Tg-Syr mice aged 8 weeks, and Il10ra and Clqtnf7 mRNAs is transiently decreased in the olfactory bulb in Tg-Syr mice aged 34 weeks and 45 weeks, respectively.

Only $I l 1 b$ and $I l 10 \mathrm{rb}$, and $\mathrm{CxCl10}$ and IllOrb mRNAs are upregulated in the cerebral neocortex and olfactory bulb, respectively, in Tg-Syr aged 80 weeks. Comparative data in the cerebral neocortex and olfactory bulb showing greater inflammatory responses in the former than in the latter, despite similar alterations regarding neuroserpin bodies in both regions, further reinforce the view that neuroserpin inclusions (as in the thalamus) are not the only determinants of inflammatory responses. Other undetermined factors may modulate the inflammatory responses.

\section{ER Stress and UPR in Tg-Syr Mice}

Because ER stress can stimulate inflammatory responses (22), we next analyzed the expression of several UPR markers in Tg-Syr mice. Protein folding at ER is modulated by 3 ER transmembrane protein sensors: PERK, inositol requiring kinase 1 (IRE1), and ATF6 (32-35). Under ER stress, PERK oligomerizes, auto-phosphorylates and phosphorylates the $\alpha$-subunit of eukaryotic initiation factor $2(\mathrm{eIF} 2-\alpha)$ at serine 51 , resulting in decreased general protein synthesis (36), but it allows specific translation of transcription factor 4 (ATF4), which promotes DNA transcription of the specific genes $\mathrm{C} /$ EBP-homologous protein $(\mathrm{CHOP})$ and asparagine synthase (ASNS) (37). Upon ER stress, ATF6 of $90 \mathrm{kDa}$ moves to the Golgi complex where it is cleaved to form the active transcription factor of $50 \mathrm{kDa}$ (ATF6-50kDa: ATF6f), which translocates to the nucleus and activates transcription of GPR78, among other stress genes (38). Finally, ER responses also activate IRE1 by dimerization and phosphorylation which, in turn, activates the transcription factor Xbox binding protein (XBP1), which activates transcription of ER-localized DnaJ 4 (ERdj4) and promotes the specific splicing of the mRNA encoding XBP1, leading to spliced XBP1 (39-41).

We found no modifications in gene expression of Gpr78, CHOP, Asns, and XBP1 in Tg-Syr mice aged 34, 45, and 80 weeks, respectively. Only Xbp1 spliced was transiently increased in Tg-Syr mice at the age of 45 weeks, and ERdJ4 at the age of 80 weeks. These results are consistent because the spliced form of Xbp1 codes for a transcription factor that induces the expression of ERdJ4. This modest and transient mRNA response is in line with the age-dependent selective and temporally limited activation of phosphorylated eIF2- $\alpha$ at the age of 20 weeks and of cleaved ATF6 protein in Tg-Syr at 34 and 45 weeks (31). Total neuroserpin increases with age and poly-neuroserpin and neuroserpin aggregates increase at the time that UPR minimizes, suggesting that transient increase in the UPR modulates mutated neuroserpin polymerization (31). The reason for transient modifications of certain UPR markers is not known but increased expression of ERdj4, a BiP (immunoglobulin-binding protein) co-chaperone, is consistent with activation of the ERAD (endoplasmic reticulum-associated degradation) pathway, which serves to remove substrates from the ER lumen and reduce cell death (4245).

\section{Links Between Failed UPR and Inflammation in Tg-Syr Mice}

Together, the present observations demonstrate discrete transient inflammatory responses and activation of the UPR at middle stages of the disease, followed by upregulation of $I l l b$ and IllOrb, and CxCl1O and Il1Orb mRNAs in the cerebral neocortex and olfactory bulb, respectively, as well as upregulation of ERdj4 in Tg-Syr aged 80 weeks. When compared with other neurodegenerative diseases, this pattern is unexpected because $\mathrm{Tg}$-Syr neuroserpinopathy is a paradigm of neurodegenerative disease with abnormal protein aggregates in the ER.

The reasons for the discrete inflammatory response may be related to the lack of extruded mutated neuroserpin polymers together with the feeble UPR in Tg-Syr mice. The UPR largely depends on the upstream chaperone glucose-regulated protein 78 (GRP78), which is located at the internal membrane of the ER and controls activation of the 3 main ER sensors: PERK, IRE1, and ATF6 (32-35). In addition, GRP94 is located in the ER lumen and acts as a chaperone of secreted and membrane proteins $(46,47)$. Double-labeling immunofluorescence and confocal microscopy disclosed colocalization of GRP78 and GRP94 and neuroserpin in the vast majority, if not all, neuroserpin inclusions in $\mathrm{Tg}$-Syr mice, at least in mice aged 80 weeks. This is not accompanied by deposition in the inclusions of other proteins involved in the UPR; thus, GRP78 and GRP94 trapping by neuroserpin aggregates in the ER seems to be selective. Based on these findings it may be suggested that GRP78 and GRP94 sequestration attenuates and distorts the UPR in Tg-Syr mouse neuroserpinopathy. Moreover, mutant neuroserpin probably does not lead to a strong activation of the UPR, because it is not really unfolded $(48,49)$.

Inclusion body isolation was performed with brain samples from FENIB patients (9) and from Tg-Portland mice (23). The protocol included homogenization of the samples followed by a detergent wash and a collagenase digestion step. After these treatments, the inclusion bodies are still intact (9); a further disruption step is necessary to release proteins that can be detected by Coomassie blue staining. In both studies, a single prominent band was observed; analysis with amino terminal sequencing or mass spectrometry revealed the band to be composed exclusively of mutant neuroserpin. However, it cannot be ruled out that other proteins (eg, mutant neuroserpin associated with chaperones not yet included or in the process of being included into polymers) are also present within inclusion bodies and are either underrepresented or under the detection limit of Coomassie blue staining, or else they are separated from the polymers during the isolation process. Whether GRP78 and GRP94 are separated during the process of subcellular fractionation is not known. Therefore, the present observations suggest that the UPR is not a crucial player in the pathogenesis of the disease and that neuroinflammatory responses are very discrete and occur at 
advanced stages of neuroserpinopathy. This is in striking contrast with other neurodegenerative diseases with abnormal protein aggregates.

\section{ACKNOWLEDGMENTS}

We wish to thank T. Yohannan for editorial assistance, and the Antibody Core Facility, University Medical Center Hamburg-Eppendorf, Hamburg, Germany, for antibody generation.

\section{REFERENCES}

1. Stoeckli ET, Lemkin PF, Kuhn TB, et al. Identification of proteins secreted from axons of embryonic dorsal-root-ganglia neurons. Eur J Biochem 1989;180:249-58

2. Osterwalder T, Contartese J, Stoeckli ET, et al. Neuroserpin, an axonally secreted serine protease inhibitor. EMBO J 1996;15:2944-53

3. Schrimpf SP, Bleiker AJ, Brecevic L, et al. Human neuroserpin (PI12): cDNA cloning and chromosomal localization to 3q26. Genomics 1997;40: 55-62

4. Hastings GA, Coleman TA, Haudenschild CC, et al. Neuroserpin, a brain-associated inhibitor of tissue plasminogen activator is localized primarily in neurons. Implications for the regulation of motor learning and neuronal survival. J Biol Chem 1997;272:33062-7

5. Osterwalder T, Cinelli P, Baici A, et al. The axonally secreted serine proteinase inhibitor, neuroserpin, inhibits plasminogen activators and plasmin but not thrombin. J Biol Chem 1998;273:2312-21

6. Krueger SR, Ghisu GP, Cinelli P, et al. Expression of neuroserpin, an in hibitor of tissue plasminogen activator, in the developing and adult nervous system of the mouse. J Neurosci 1997;17:8984-96

7. Galliciotti G, Sonderegger P. Neuroserpin. Front Biosci 2006;11:33-45

8. Davis RL, Shrimpton AE, Holohan PD, et al. Familial dementia caused by polymerization of mutant neuroserpin. Nature 1999;40:1376-9

9. Davis RL, Holohan PD, Shrimpton AE, et al. Familial encephalopathy with neuroserpin inclusion bodies. Am J Pathol 1999;155:1901-13

10. Takao M, Benson MD, Murrell JR, et al. Neuroserpin mutation S52R causes neuroserpin accumulation in neurons and is associated with progressive myoclonus epilepsy. J Neuropathol Exp Neurol 2000;59:1070-86

11. Bradshaw CB, Davis RL, Shrimpton AE, et al. Cognitive deficits associated with a recently reported familial neurodegenerative disease: Familial encephalopathy with neuroserpin inclusion bodies. Arch Neurol 2001;58:1429-34

12. Yazaki M, Liepnieks JJ, Murrell JR, et al. Biochemical characterization of a neuroserpin variant associated with hereditary dementia. Am J Pathol 2001;158:227-33

13. Davis RL, Shrimpton AE, Carrell RW, et al. Association between conformational mutations in neuroserpin and onset and severity of dementia. Lancet 2002;359:2242-7

14. Gourfinkel-An I, Duyckaerts C, Camuzat A, et al. Clinical and neuropathologic study of a French family with a mutation in the neuroserpin gene. Neurology 2007;69:79-83

15. Coutelier M, Andries S, Ghariani S, et al. Neuroserpin mutation causes electrical status epilepticus of slow-wave sleep. Neurology 2008;71:64-6

16. Hagen MC, Murrell JR, Delisle MB, et al. Encephalopathy with neuroserpin inclusion bodies presenting as progressive myoclonus epilepsy and associated with a novel mutation in the Proteinase Inhibitor 12 gene. Brain Pathol 2011;21:575-82

17. Davis RL, Collins GH. Familial encephalopathy with neuroserpin inclusion bodies. In: Dickson DW, Weller RO, eds. Neurodegeneration: The Molecular Pathology of Dementia and Movement Disorders. Oxford, UK: Wiley-Blackwell 2011: 456-60

18. Miranda E, MacLeod I, Davies MJ, et al. The intracellular accumulation of polymeric neuroserpin explains the severity of the dementia FENIB. Hum Mol Genet 2008; 17:1527-39

19. Whisstock JC, Bottomley SP. Structural biology: Serpins' mystery solved. Nature 2008;455:1189-90

20. Zhang Q, Law RH, Bottomley SP, et al. A structural basis for loop Csheet polymerization in serpins. J Mol Biol 2008;376:1348-59
21. Yamasaki M, Li W, Johnson DJ, et al. Crystal structure of a stable dimer reveals the molecular basis of serpin polymerization. Nature 2008;455: 1255-8

22. Zhang K, Kaufman RJ. From endoplasmic-reticulum stress to the inflammatory response. Nature 2008;454:455-62

23. Galliciotti G, Glatzel M, Kinter J, et al. Accumulation of mutant neuroserpin precedes development of clinical symptoms in familial encephalopathy with neuroserpin inclusion bodies. Am J Pathol 2007;170:1305-13

24. Takasawa A, Kato I, Takasawa K, et al. Mutation-, aging-, and gene dosage-dependent accumulation of neuroserpin (G392E) in endoplasmic reticula and lysosomes of neurons in transgenic mice. J Biol Chem 2008; 283:35606-13

25. Takano K, Kitao Y, Inagi R, et al. A rat model of human FENIB (familial encephalopathy with neuroserpin inclusion bodies). Biochem Biophys Res Commun 2006;346:1040-7

26. Llorens F, López-González I, Thüne K, et al. Subtype and regional-specific neuroinflammation in sporadic Creutzfeldt-Jakob disease. Front Aging Neurosci 2014;6:198

27. López-González I, Schlüter A, Aso E, et al. Neuroinflammatory signals in Alzheimer disease and APP/PS1 transgenic mice: Correlations with plaques, tangles, and oligomeric species. J Neuropathol Exp Neurol 2015;74:319-44

28. López-González I, Aso E, Carmona M, et al. Neuroinflammatory gene regulation, mitochondrial function, oxidative stress and brain lipid modifications with disease progression in tau P301S transgenic mice as a model of FTLD-tau. J Neuropathol Exp Neurol 2015;74:975-99

29. Koch-Nolte F, Glowacki G, Bannas P, et al. Use of genetic immunization to raise antibodies recognizing toxin-related cell surface ADP-ribosyltransferases in native conformation. Cell Immunol 2005;236:66-71

30. Zamarbide M, Martinez-Pinilla E, Ricobaraza A, et al. Phenyl acyl acids attenuate the unfolded protein response in tunicamycin-treated neuroblastoma cells. PLoS One 2013;8:e71082

31. Schipanski A, Oberhauser F, Neumann M, et al. Lectin OS-9 delivers mutant neuroserpin to endoplasmic reticulum associated degradation in familial encephalopathy with neuroserpin inclusion bodies. Neurobiol Aging 2014;35:2394-2403

32. Schröder M, Kaufman RJ. The mammalian unfolded protein response. Annu Rev Biochem 2005;74:739-89

33. Kohno K. How transmembrane proteins sense endoplasmic reticulum stress. Antioxid Redox Signal 2007;9:22

34. Schröder M. Endoplasmic reticulum stress responses. Cell Mol Life Sci 2008;65:862-94

35. Bernales S, Morales-Soto M, McCullagh E. Unfolded protein stress in the endoplasmic reticulum and mitochondria: A role in neurodegeneration. Front Aging Neurosci 2012;4:1-13

36. Donnelly N, Gorman AM, Gupta S, et al. The eIF2 $\alpha$ kinases: their structure and functions. Cell Mol Life Sci 2013;70:3493-511

37. Ron D, Walter P. Signal integration in the endoplasmic reticulum unfolded protein response. Nat Rev Mol Cell Biol 2007;8:519-29

38. Chen X, Shen J, Prywes R. The luminal domain of ATF6 senses endoplasmic reticulum (ER) stress and causes translocation of ATF6 from the ER to the Golgi. J Biol Chem 2002;277:13045-52

39. Sidrauski C, Walter P. The transmembrane kinase Ire1p is a site-specific endonuclease that initiates mRNA splicing in the unfolded protein response. Cell 1997;90:1031-39

40. Yoshida H, Matsui T, Yamamoto A, et al. XBP1 mRNA is induced by ATF6 and spliced by IRE1 in response to ER stress to produce a highly active transcription factor. Cell 2001;107:881-91

41. Calfon M, Zeng H, Urano F, et al. IRE1 couples endoplasmic reticulum load to secretory capacity by processing the XBP-1 mRNA. Nature 2002; 415:92-6

42. Kurisu J, Honma A, Miyajima H, et al. MDG1/ERdj4, an ER-residen DnaJ family member, suppresses cell death induced by ER stress. Genes Cells 2003;8:189-202

43. Berger BJ, Müller TS, Buschmann IR, et al. High levels of the molecular chaperone Mdg1/ERdj4 reflect the activation state of endothelial cells. Exp Cell Res 2003;290:82-92

44. Lai CW, Otero JH, Hendershot LM, et al. ERdj4 protein is a soluble endoplasmic reticulum (ER) DnaJ family protein that interacts with ER-associated degradation machinery. Biol Chem 2012;287:7969-78

45. Fritz JM, Dong M, Apsley KS, et al. Deficiency of the BiP cochaperone ERdj4 causes constitutive endoplasmic reticulum stress and metabolic defects. Mol Biol Cell 2014;25:431-40 
46. Little E, Ramakrishnan M, Roy B, et al. The glucose-regulated proteins (GRP78 and GRP94): Functions, gene regulation, and applications. Crit Rev Eukaryot Gene Expr 1994;4:1-18

47. Marzec M, Eletto D, Argon Y. GRP94: An HSP90-like protein specialized for protein folding and quality control in the endoplasmic reticulum. Biochim Biophys Acta 2012;1823:774-87
48. Kroeger H, Miranda E, MacLeod I, et al. Endoplasmic reticulum-associated degradation (ERAD) and autophagy cooperate to degrade polymerogenic mutant serpins. J Biol Chem 2009;284:22793-802

49. Davies MJ, Miranda E, Roussel BD, et al. Neuroserpin polymers activate NF-kappaB by a calcium signaling pathway that is independent of the unfolded protein response. J Biol Chem 2009;284:18202-9 